\title{
The current role of glucose control medications in primary and secondary prevention for cardiovascular disease
}

\begin{abstract}
Except for glucose management, prevention of CVD Follows the same general principles as those for people without DM. The goal of this manuscript is to review the pharmacological strategies aimed at controlling blood glucose that were proven to be useful to prevent death related to CVD.

The importance of intensive glucose lowering with respect to micro vascular risk complications in newly diagnosed patients with DM has been well established. The UKPDS 34 Trial supported the systematic and current use of metformin as a first-line drug therapy for patients with DM and established the great importance of intensive glucose lowering with this drug in order to promote CVD risk reduction in patients with DM.

The recent use of glucagon-like peptide 1 analogues to treat diabetic patients had controversial results with a worse evolution of heart failure patients but had a better renal prognosis in non heart failure patients.

New anti diabetic drugs can also be used to reduce the incidence of future cardiovascular events. Two SGLT2 inhibitors (Empaglifozin and Canaglifozin) have lessened CV???? Mortality and HF in high risks patients with prior CVD or with a high cardiovascular risk

Early evidence suggests no CVD benefit with short-term use of dipeptidyl peptidase 4 (DPP-4) inhibitors in people at high risk for CVD. Pioglitazone can also be useful to reduce the incidence of myocardial infarctions and stroke, but did not have an impact on total mortality in patients without established DM.

There is no doubt about the importance of intensive glucose lowering (only reached with the use of Insulin) in patients with type 1 DM. Further studies are needed on metformin and GLP-1 receptor agonists in patients with type 1 DM to determine whether they improve glycemic control, aid in weight reduction and improve clinical outcomes.
\end{abstract}

Keywords: cardiovascular mortality, cardiovascular prevention, diabetes mellitus, cardiovascular risk
Volume 2 Issue 2 - 2017

\author{
João Lucas O Connell,' Gabriela Carolina \\ Borges, ${ }^{2}$ Ana Carolina Gondin, ${ }^{2}$ Leonardo \\ Roever $^{2}$ \\ 'Department of Cardiology, Federal University of Uberlândia, \\ Brazil \\ ${ }^{2}$ Federal University of Uberlândia, Brazil
}
Correspondence: Joao Lucas O Connell, Department of Cardiology, Federal University of Uberlandia, Brazil, Tel 5534 99665-I I59, ZIP CODE 384II-I5I,

Email oconnelljl@icloud.com

Received: August 24, 2017 | Published:September 19, 2017

\section{Introduction}

In most developed countries cardiovascular diseases (CVD) remain the main cause of morbidity and mortality in the general population, especially among the elderly. ${ }^{1}$ CVD causes 18 million deaths per year globally and a similar number of nonfatal cardiovascular events. ${ }^{2}$ People with Diabetes mellitus (DM) are on average at double the risk of CVD. ${ }^{3}$ Cardiovascular disease (CVD) prevention, i.e. coronary heart disease, stroke, and peripheral artery disease, includes all the actions intended to eliminate or reduce the impact of these disorders and their disabilities in the population or even targeted at individuals.

Except for glucose management, prevention of CVD follows the same general principles as those for people without DM. Achieving low BP levels and low LDL-C and total cholesterol concentrations are particularly important. Many treatment targets are more stringent for patients with DM. ${ }^{4}$ Typically patients with type 2 DM (T2DM) have multiple CVD risk factors, each requiring treatment according to existing guidelines. The goal of this article is to review the pharmacological strategies aimed to control blood glucose that were proven to be useful in preventing death related to CVD.

\section{Discussion}

Biguanides have many theoretical benefits over other agents in the treatment of T2DM including reducing hepatic gluconeogenesis, decreasing plasma insulin levels, and facilitating weight loss. However, the biguanide phenformin was associated with increased CV and all-cause mortality in UGDP (1975). ${ }^{5}$

In the UK, Prospective Diabetes Study 33 (UKPDS 33) established the importance of intensive glucose lowering with respect to micro vascular risk complications in patients newly diagnosed with DM. In this important and historical trial, intensive blood glucose control by either sulphonylureas or insulin substantially decreased the risk of micro vascular complications, but not macro vascular disease, in type 2 diabetic patients. None of the individual drugs had an adverse effect on cardiovascular outcomes. All intensive treatment increased the risk of hypoglycemia. ${ }^{6}$ 
Published simultaneously with the above, the UKPDS 34 Trial supported the systematic and current use of metformin as a first-line drug therapy for patients with DM and established the great importance of intensive glucose lowering with this drug in order to promote CVD risk reduction in patients with DM. In this important and historical trial 1,704 overweight patients with newly diagnosed T2DM were randomized to one of three arms: conventional therapy with diet alone, intensive therapy with metformin, or intensive therapy with an earlygeneration of antiglycemic agents (chlorpropamide, glibenclamide, or insulin). The primary analysis compared metformin to diet alone, with a secondary analysis comparing metformin to intensive therapy with the other agents. With a median follow-up of 10.7 years, metformin was associated with a reduction in DM-related complications and in all-cause mortality when compared to the other two arms of therapy. These benefits persisted for an additional 10 years of follow-up. These studies form the basis for the early administration of metformin in overweight patients with T2DM.

The benefits of metformin monotherapy in overweight patients with T2DM was further solidified by a 2005 Cochrane review of 29 randomized trials comparing metformin to conventional therapy with diet or other modern antiglycemic agents. This meta-analysis demonstrated a benefit for metformin monotherapy across a diverse range of outcomes including glycemic control, weight loss, lipid and blood pressure control, diabetes-related mortality, all-cause mortality, and incidence of myocardial infarction.

Since the publication of UKPDS 34, several generations of antiglycemic agents have been developed, and direct comparisons with metformin have been performed both prospectively and retrospectively. The large multicenter Spread-Dimcad trial (2013) trial (2013) randomized patients with T2DM and CAD to either metformin and glipizide for 3 years. At a median follow-up of 5 years, both groups achieved goal $\mathrm{HbAlc}$ levels $(7.1 \%$ vs. $7.0 \%)$ but metformin was associated with a $12 \%$ absolute risk reduction in the composite primary outcome of nonfatal MI, nonfatal stroke, revascularization, CV mortality, or all-cause mortality. ${ }^{10}$

Recent trials using newer therapies like dipeptidyl peptidase-4 demonstrated non inferiority (safety) in cardiovascular outcomes among patients with T2DM and existing CVD or at higher risk of events. ${ }^{11-14}$ Recently, for example, the use of sitagliptin in more than 7.000 patients - in the TECOS trial - was not associated with an increased risk of heart failure or related adverse outcomes after Sitagliptin therapy.11 There was, however, an increase in the rate of hospitalization for HF with the use of saxagliptin (SAVOR-TIMI 53) trial. $^{14}$

The glucagon-like peptide 1 analogue is another type of antidiabetic drugs that had controversial results in outcomes among diabetic patients in recent trials. The use of Liraglutide in patients with high-risk heart failure patients with reduced ejection fraction (FIGHT) was associated with an almost 30\% increase in the rates of death or heart failure hospitalization within 6 months. ${ }^{15}$ Very recently, the use of the same GLP1 analogue in type 2 diabetic patients without heart failure was associated with lower rates of the development and progression of diabetic kidney disease than placebo. ${ }^{16}$ These results suggests that the use of this type of anti-diabetic drugs could be associated with less future kidney aggressions (manifested by a lower rate of micro albuminuria), but with higher rates of hospitalization or death in patients with severe heart failure.
More recently, a newer type of anti diabetic drug has shown incredibly good results for secondary prevention of new cardiovascular events in diabetic patients: the Sodium-glucose co-transporter-2 inhibitors (SGLT2i). Empaglifozin, one of these SGLT2i, was the first to demonstrate unequivocal benefit in the cardiovascular outcome of diabetic patients with a history of a prior cardiovascular event (heart attack, stroke or others). In the EMPAREG OUTCOME trial, 7020 patients were randomized to use Empaglifozin added to standard antidiabetic treatment or standard treatment alone. Patients using this SGLT2i presented a $38 \%$ reduction in CVD death, all-cause mortality $(32 \%)$ and also in the hospitalization for heart failure $(35 \%)$. In this study, the results of non-fatal myocardial infarction and stroke were not reduced. This fact, added to the rapid separation of mortality curves, suggests that the mechanism of benefit was likely to relate more to cardio-renal haemodynamic effects than to atherothrombotic actions or effects of glucose lowering per se. ${ }^{17}$

The use of Canagliflozin (another type of SGLT2i) was also related to the reduction of cardiovascular and renal events in type 2 diabetic patients. This time, this type of drug was used for primary (not secondary) prevention of cardiovascular events. The CANVAS Program integrated data from two trials involving a total of 10,142 participants with type 2 diabetes and high cardiovascular risk. Participants in each trial were randomly assigned to receive Canagliflozin or placebo and were followed for a mean of 188.2 weeks. The primary outcome was a composite of death from cardiovascular causes, nonfatal myocardial infarction, or nonfatal stroke. Patients treated with Canaglifozin, had a significantly lower risk (14\%) of death from cardiovascular causes, nonfatal myocardial infarction, or nonfatal stroke than those who received placebo, although this treatment led to a greater risk of peripheral amputation (primarily at the level of the toe or metatarsal: 1,97 times more events) and infection of male genitalia (3,2 times more events). ${ }^{18}$ Another recently published meta-analyses (The CVDReal study) also showed lower rates of hospitalization for heart failure $(39 \%)$ and all cause mortality (51\%) in new users of three different SGLT2i (predominantly Canaglifozin and Dapaglifozin and a lower proportion of Empaglifozin) when compared with standard glucose treatment with the use of other types of medication for diabetes mellitus. ${ }^{19}$

Another anti diabetic drug that also demonstrated some impact in the reduction of new cardiovascular events that we must highlight in this summary was Pioglitazone. In the IRIS Trial (a multicenter, double-blind trial), 3876 patients who had had a recent ischemic stroke or transient ischemic attack (TIA) were randomly assigned to receive either pioglitazone (target dose of $45 \mathrm{mg}$ daily) or placebo. At the time of randomization, eligible patients did not yet have the diagnosis of diabetes but were found to have insulin resistance (a high HOMA-IR index). The primary outcome was fatal or nonfatal stroke or myocardial infarction. By 4.8 years, a primary outcome had occurred in 175 of 1939 patients $(9.0 \%)$ in the pioglitazone group and in 228 of $1937(11.8 \%)$ in the placebo group (hazard ratio in the pioglitazone group, $0.76 ; 95 \%$ confidence interval $[\mathrm{CI}], 0.62$ to $0.93 ; \mathrm{P}=0.007)$. Pioglitazone was also associated with a lower risk of diabetes but with higher risks of weight gain, edema, and fracture. Although there was an evident reduction in the incidence of non fatal stroke or myocardial infarction, there was no significant reduction in group difference in all-cause mortality (hazard ratio, 0.93 ; 95\% CI, 0.73 to $1.17 ; \mathrm{P}=0.52) .^{20}$ 
The results of the IRIS trial are in contrast to the findings of two trials involving patients with type 2 diabetes. In the Prospective Pioglitazone Clinical Trial in the Macrovascular Events (PROactive) trial $^{21,22}$ the rate of primary outcome of death, myocardial infarction, stroke, acute coronary syndrome, vascular surgery, and amputation was not significantly lower among patients in the pioglitazone group than among those in the placebo group. In the Bypass Angioplasty Revascularization Investigation 2 Diabetes (BARI-2D) trial, ${ }^{23}$ the rate of primary outcome of death, stroke, or myocardial infarction was not significantly lower among patients receiving rosiglitazone and metformin (insulin-sparing strategy) than among those receiving insulin and sulfonylurea therapy (insulin-providing strategy). However, the IRIS results are consistent with the findings regarding a secondary outcome in the PROactive trial (i.e., that the rates of death, myocardial infarction, or stroke were significantly lower with pioglitazone than with placebo) and with the findings of trials showing a favorable effect of pioglitazone on the progression of subclinical atherosclerosis among patients with or without diabetes. ${ }^{20,24-26}$

Finally, we must remember that The Diabetes Control and Complications Trial (DCCT) established the importance of tight glucose control to lessen the risks of both micro vascular and macro vascular disease in type $1 \mathrm{DM}$ patients. A 27 year follow-up of this trial showed that 6.5 years of initial intensive DM therapy in type $1 \mathrm{DM}$ was associated with a modestly lower all-cause mortality rate when compared with conventional therapy. ${ }^{27}$ A glycemic target for $\mathrm{HbA} 1 \mathrm{c}$ of $6.5-7.5 \%(48-58 \mathrm{mmol} / \mathrm{mol})$ appears to be a balanced approach for long-term care of patients with type $1 \mathrm{DM}$. Within patients with type 1 Diabetes, the use of other insulin analogues, insulin pumps and continuous glucose monitoring to improve glycemic control and mortality while minimizing hypoglycemia and other side effects is the subject of actual intense research. At the same time, there is still insufficient data to affirm that there is any benefit in the use of other oral agents (e.g. metformin, GLP-1 agonists), commonly used in type $2 \mathrm{DM}$, in type 1 patients.

\section{Conclusion}

Effective control of blood glucose levels has been proven to prevent the development of cardiovascular disease. In patients with type 2 diabetes mellitus, several studies have established the importance of glucose lowering to prevent CVD, with the best evidence supporting metformin, leading to its position as first-line therapy in the treatment of this disease, especially because of its good cardiovascular safety. New anti diabetic drugs can also be used to reduce the incidence of future cardiovascular events. Two SGLT2 inhibitors (Empaglifozin and Canaglifozin) have lessened CV mortality and HF in high risk patients with prior CVD or with a high cardiovascular risk. More research on the benefits of glucagon-like peptide 1 (GLP-1) receptor agonists on CVD risk is needed and trials are due to be reported in subsequent years. Early evidence suggests no CVD benefit with short-term use of dipeptidyl peptidase 4 (DPP-4) inhibitors in people at high risk for CVD. Pioglitazone can also be useful to reduce the incidence of myocardial infarctions and stroke, but did not affect total mortality in patients without established DM. There is no doubt about the importance of intensive glucose lowering (only reached with the use of Insulin) in patients with type $1 \mathrm{DM}$. Further studies are needed on metformin and GLP-1 receptor agonists in patients with type $1 \mathrm{DM}$ to determine whether they improve glycemic control, help in weight reduction or improve clinical outcomes.

\section{Acknowledgments}

The authors state that they have no acknowledgments to make.

\section{Conflicts of interest}

Authors declare there is no conflict of interest in composing this manuscript.

\section{References}

1. Piepoli MF, Hoes AW, Agewall S, et al. European Guidelines on cardiovascular disease prevention in clinical practice: The Sixth Joint Task Force of the European Society of Cardiology and Other Societies on Cardiovascular Disease Prevention in Clinical Practice (constituted by representatives of 10 societies and by invited experts). Eur Heart J. 2016;37(29):2315-2381.

2. GBD 2013 Risk factors Collaborators. Global, regional, and national comparative risk assessment of 79 behavioral, environmental and occupational, and metabolic risks of clusters of risks in 188 countries, 1990-2013: a systematic analysis for the Global Burden of Disease Study. Lancet. 2015;386(10010):2287-2323.

3. Sarwar N, Gao P, Seshasai SR, et al. Diabetes mellitus, fasting blood glucose concentration, and risk of vascular disease: a collaborative metaanalysis of 102 prospective studies. Lancet. 2010;375(9733):2215-2222.

4. Piepoli MF, Hoes AW, Agewall S, et al. European Guidelines on cardiovascular disease prevention in clinical practice: The Sixth Joint Task Force of the European Society of Cardiology and Other Societies on Cardiovascular Disease Prevention in Clinical Practice (constituted by representatives of 10 societies and by invited experts) Developed with the special contribution of the European Association for Cardiovascular Prevention \& Reabilitation (EACPR). Eur Heart J. 2016;37(29):2315-2381.

5. University Group Diabetes Program. A study of the effects of hypoglycemic agents on vascular complications on patients with adult onset diabetes: V-evaluation of phenformin therapy. Diabetes. 1975;24 supp1 1:65-184.

6. Turner R. Intensive blood-glucose control with sulphonylureas or insulin compared with conventional treatment and risk of complications in patients with type 2 diabetes. Lancet. 1998;352(9131):837-853.

7. UKPDS Study Group. Effect of intensive blood-glucose control with metformin on complications in overweight patients with type 2 diabetes (UKPDS 34). UK Prospective Diabetes Study (UKPDS) Group. Lancet. 1998;352(9131):854-865.

8. Holman RR, Paul SK, Bethel MA, et al. 10-year follow-up of intensive glucose control in type 2 diabetes. NEngl JMed.2008;359(15):1577-1589.

9. Saenz A1, Mataix A, Ausejo M, et al. Metformin monotherapy for type 2 diabetes mellitus. Cochrane Database Syst Rev. 2005;(3):CD002966.

10. Hong J, Zhang Y, Lai S, et al. Effects of metformin versus glipizide on cardiovascular outcomes in patients with type 2 diabetes and coronary artery disease. Diabetes Care. 2013;36(5):1304-1311.

11. Green JB, Bethel MA, Armstrong PW, et al. Effect of sitagliptin on cardiovascular outcomes in type 2 diabetes. $N$ Engl $\mathrm{J} \mathrm{Med}$. $2015 ; 373: 232-242$

12. Pfeffer MA, Claggett B, Diaz R, et al. Lixisenatide in patients with type 2 diabetes and acute coronary syndrome. $N$ Engl $\mathrm{J} \mathrm{Med}$. 2015;373:2247-2257.

13. White WB, Cannon CP, Heller SR, et al. Alogliptin after acute coronary syndrome in patients with thpe 2 diabetes. $N$ Engl $\mathrm{J} \mathrm{Med}$. 2013;369:1327-1335. 
14. Scirica BM, Bhatt DL, Braunwald E, et al. Saxagliptin and cardiovascular outcomes in patients with type 2 diabetes mellitus. $N$ Engl $\mathrm{J} \mathrm{Med}$. 2013;369:1317-1326.

15. Margulies KB, Anstrom KJ, Redfield MM, et al. A randomized trial of Liraglutide for high-risk heart failure patients with reduced ejection fraction (FIGHT). American Heart Association 2015 Scientific Sessions. Orlando Florida, USA; 2015.

16. Johannes FE, Mann MD, Orsted DD, et al. Liraglutide and renal outcomes in type 2 Diabetes. N Engl J Med. 2017;377(9):839-848.

17. Zinman B, Wanner C, Lachin JM, et al. Empagliflozin, cardiovascular outcomes, and mortality in type 2 diabetes. $N$ Engl J Med. 2015;373(22):2117-2128.

18. Neal B, Perkovic V, Mahaffey K, et al. Canagliflozin and Cardiovascular and Renal Events in Type 2 Diabetes. N Engl J Med. 2017;377:644-657.

19. Kosiborod M, Matthew AC, Alex ZF, et al. Lower Risk of Heart Failure and Death in Patients Initiated on SGLT-2 Inhibitors Versus Other Glucose-Lowering Drugs: The CVD-REAL Study. Circulation. $2017 ; 1-117$

20. Kernan W, Viscoli C, Furie L, et al. Pioglitazone after Ischmic Stroke or Transient Ischemic Attack. N Engl J Med. 2016;374:1321-1331.

21. Wilcox R, Bousser MG, Betteridge DJ, et al. Effects of pioglitazone in patients with type 2 diabetes with or without previous stroke: results from PROactive (PROspective pioglitAzone Clinical Trial In macroVascular Events 04. Stroke. 2007;38(3):865-873.
22. Dormandy JA, Charbonnel B, Eckland DJA, et al. Secondary prevention of macrovascular events in patients with type 2 diabetes in the PROactive Study (PROspective pioglitAzone Clinical Trial In macroVascular Events): a randomised controlled trial. Lancet. 2005;366(9493):1279-1289.

23. The BARI 2D Study Group. A randomized trial of therapies for type 2 diabetes and coronary artery disease. NEngl J Med. 2009;360:2503-2515.

24. Nissen SE, Nicholls SJ, Wolski K, et al. Comparison of pioglitazone vs glimepiride on progression of coronary atherosclerosis in patients with type 2 diabetes: the PERISCOPE randomized controlled trial. JAMA. 2008;299(13):1561-1573.

25. Mazzone T, Meyer PM, Feinstein SB, et al. Effect of pioglitazone compared with glimepiride on carotid intima-media thickness in type 2 diabetes: a randomized trial. JAMA. 2006;296(21):2572-2581.

26. Saremi A, Schwenke DC, Buchanan TA, et al. Pioglitazone slows progression of atherosclerosis in prediabetes independent of changes in cardiovascular risk factors. Arterioscler Thromb Vasc Biol. 2013;33(2):393-399.

27. Orchand TJ, Nathan DM, Zinman B, et al. Association between 7 years of intensive treatment of type 1 diabetes and long-term mortality. JAMA. $2015 ; 313(1): 45-53$. 